\title{
Research on the Claim of Duration and Cost Based on the Model of Different Engineering Contracts
}

\author{
Meijing Wang \\ School of Management, Tianjin University of Technology, Tianjin 300384, China \\ JessicaMJ369@163.com
}

Keywords: The Belt and Road, Engineering Claims, Construction.

\begin{abstract}
In the "The Belt and Road” vision, Chinese engineering develops rapidly in foreign country. Engineering claims and their management have been the weak links in the field of engineering construction, mainly reflected in the legal system is not perfect, the claim consciousness is weak, the lack of experience in construction management, claims the lack of specialized personnel and etc. To solve this problem, first, this paper analyzed the claim of duration and cost in the determination of the circumstances. Then according to the comparative analysis of different forms of contract claims on project, in order to help Chinese contractor to select a reasonable contracts in foreign engineering, and enhance the sense of claims. After the occurrence of the claim, a reasonable claim can be made according to the agreement.
\end{abstract}

\section{Introduction}

With the continuous progress and development of the process of economic globalization, the prospect of international construction market is wider. Meanwhile, the claim problem of construction projects is emerging with the development of engineering market. A large number of engineering practices have shown that engineering claims and their management have been the weak links in the field of engineering construction. It is found that scholars have many researches on engineering claims under the conditions of FIDIC contracts, but there is a lack of comprehensive and systematic comparative study of claims for other engineering contract models and the claims mechanism of different engineering contracts. In view of this, according to the international common engineering contract "Conditions of Contract for Civil Engineering Construction ", " Conditions of Contract for NEC" and "Conditions of Contract for AIA" and the "Contract for Works of Engineering Construction " (GF-2013-0201) as the research object, Through the analysis method of research theory and contrastive based on the claims of cost and duration, this topic in-depth analysis of project contract different templates. Finally, through the in-depth analysis and understanding of the claim mechanism under the model of different engineering contracts, it can strengthen the Contractor's claim consciousness and standardize the claim operation.

\section{Related Research on the Claim of Duration and Cost}

\subsection{Related Research on the Claim of Duration}

In the construction process, the project construction period is relatively long. Unpredictable factors, interference factors of construction is also inevitable. It often makes the schedule cannot be achieved, resulting in delays and leading to claims. This is one of the most common problems in engineering construction field. For a contractor, the claim of duration is the key point for the contractor to claim the project successfully. The contractor not only needs to know the cause of the claim of duration for the project, but also needs to know clearly the procedure of the claim of duration for the construction period and the calculation of the extension time of the construction period. At present, the theoretical research on the claim in China can be divided into two parts: the qualitative analysis and the quantitative analysis of the claim for the period of work. Liao Wei[1], Wang Ruiqing, Hou Xiu[2] and other scholars' research belongs to qualitative research. By studying the contract terms, this paper analyzes the reason, basis and classification of claim for duration. Study on the quantitative study of Dai Qiongying[3], Xiong Xuhong and Li Quanrong[4] and other scholars. Quantitative research and 
analysis of the related calculation methods of engineering claims, such as dynamic analysis, proportion method and right compensation method, will make the claim more specific.

From the point of view of the research, the related researches are mainly concentrated on four aspects: the contract terms of claims, the identification of claims events, the principles and procedures of claims for contractors, and the calculation method of claims

\subsection{Related Research on the Claim of Cost}

A claim for cost refers to the economic loss or extra expenses that the contractor demands to the owner for compensation that should not be borne by the contractor himself. When the contractor claims to the owner, besides the claim for duration, the cost claim is also a key factor, and the claim for expenses affects the income of the contracted project of the contractor. Research by combing different scholars for cost claims, He Bosen, Zhang Shuibo[5] and other scholars is mainly based on the FIDIC contract, analysis of time claims and cost claims and its calculation method, Shan Jian[6] and other scholars introduced the system of limitation of claim, and puts forward the method of duration of claims, such as dynamic analysis method, ratio method and right compensation method. Liang Hongsheng[7] and other scholars have analyzed the calculation method of the cost claim value, which provides the basis for the calculation of the claim for construction delay costs.

\section{Determination of the Claims of Duration and Cost}

When the claim is caused by the owner or the factors that are not controlled by both parties, the claim mainly includes two aspects: the request for the extension of duration and the demand for economic compensation. These two requirements are sometimes separated, sometimes at the same time. This paper will study the claim of duration and the claim of the cost respectively.

\subsection{The Determination of the Claim of Duration}

The claim of duration is the Contractor's claim to the rights. It is the Contractor to avoid the responsibility which caused by delay of duration. Once the claim is approved, the contractor can not only exempt the tardiness from the liability for breach of contract, but also may obtain economic compensation.

The delay of construction period is likely to cause great losses to both sides. Therefore, grasping the content and mechanism of the claim for time limit, reasonably dividing the responsibilities, identifying the claim opportunities and filing claims application is the guarantee for the successful implementation of the claim for time limit.

1. The classification of delays in duration

Duration delay is mainly divided into forgiving delay and unforgivable delay. Delays due to the responsibility of the owner or the objective cause are pardon. Forgivable delay is divided into two kinds: one is forgivable and compensable, the other is forgivable and uncompensable. The claim to be forgiven for the delay is caused by the owner or the engineer, and the contractor may claim for the time and cost. Once it occurs. Forgiveness cannot be compensated for by the objective reasons that are not attributable to both parties, so the contractor cannot ask for the cost compensation and may require proper delay in the duration.

2. The procedure of delay in duration

After summarizing and analyzing the claims of the contractor for the duration of the project, the basic operation procedure can be summarized as follows:

First, we must make clear the cause of the delay in the construction period. Only when the delay caused by the reason of non-contractor is determined, can the contractor have the right to ask the owner for the corresponding claim.

Secondly, we use the network plan method reasonably to plan and analyze the delay event and determine whether it happens on the critical path. If the delay occurs on the critical path, the contractor can claim it, otherwise it cannot.

In the end, whether the claim can be made. The network plan method is also used to determine whether the delay is caused by the owner's reason. 


\subsection{The Determination of the Claim of Cost}

The project claim mainly refers to the economic compensation [8].In the engineering practice, if the claim happened, the contractor puts forward the claim of duration, the purpose is to obtain economic compensation or reduce the economic loss. As long as the owner's approval is obtained, the claim can be carried out and the liability for compensation for the period of disoperation may be exempted. If the schedule in advance, the contractor may also put forward a claim due to the cost of crashing caused by the contractor not only saves time, but also get the corresponding economic compensation, therefore, claims to a certain extent, but also to the cost claim.

\section{Comparison and Analysis of Duration and Cost Claims Under Different Project Contract Models}

Under the different model of the contract, the claim for the duration and cost caused by the owner's cause and the claim for the time and cost of the two parties are not imputable. In different model of the contracts, finds the commonality and individuality of the claims dealing with the claims.

\subsection{Claim Caused by Owner's Reason}

1. The common points in the treatment of claims caused by owners in different engineering contracts are manifested in the following:

(1) The error caused by drawings is changed or delayed. The stipulation of the claim is basically the same, that is, under the instruction of the engineer, the contractor only calculates the downtime losses of the affected mechanical equipment.

(2) The project change leads to the increase of the time limit for the construction period. The contractor cannot finish the project on schedule. The owner only needs to delay the time limit, and does not need the corresponding cost compensation

2. Different project contracts have different points of claim for owners' reasons, which are specifically reflected in the cost calculation.

The construction project construction contract and the FIDIC contract are calculated according to the lease or depreciation rate when calculating the loss caused by mechanical equipment shutdown. When the NEC engineering contract adjusts the cost of the construction equipment, the cost of the adjustment is negotiated by the employer and the contractor.

\subsection{Claim for No Responsibility Between the Two Parties}

1. The common points in the handling of claims that are not clear to both parties in different contracts are manifested in the following:

(1) In the period of engineering change leads to increased, the contractor cannot be delivered on time, without the payment of tardiness default loss compensation.

(2) Due to the downtime caused by bad weather and the delay in the time of the work, the Contractor shall take the responsibility for the delay, and the duration of the work is postponed according to the actual conditions.

2. The differences in handling claims that are not clear to both parties in different contracts are manifested in the following:

(1) The claim caused by bad weather. The construction project contract of construction and the AIA contract do not pay the additional cost. The FIDIC contract does not support the claim for the Contractor's losses caused by bad weather. The NEC contract clearly stipulates that the recorded meteorological data and meteorological data indicate that the average frequency of the value is less than ten years, which is a compensation event. Besides compensating the construction period, it will also compensate for a certain cost.

(2) The claim caused by economic risk. The "model of construction project contract" and the FIDIC contract mainly consider the risks caused by inflation. There is a clear provision in the NEC contract that the inflation factor is lower than the actual inflation rate. But there is no inflation rule in the AIA. 


\section{Conclusion}

Through the analysis of the claim for duration and the claim for cost under different engineering contract models, it can be seen that the claim for duration and the claim for cost always happen together, and different engineering contracts have different conventions. The model of the contract of construction stipulates and compensation that the contractor can get when different claim events happen, but the specific compensation ways of different contract models are different. Therefore, in engineering practice, we should consider the actual situation of the host country, choose the contract template reasonably, and make reasonable claim according to the agreement after the claim incident happens.

\section{References}

[1]. Liao wei. A brief discussion on the claim and claim for the construction period in the international project contract. The construction of science and technology in Fujian, 1999, (04) :40 41.

[2]. Wang Ruiqing, Hou Xiu. FIDIC claim for project delay under contract conditions. Shanxi construction 2000, (06):92 93.

[3]. Dai Qiongying .The calculation of the cost and duration of claims in FIDIC "Conditions of Contract for Engineering Construction ". Sichuan building materials, 2010, (03):271 272.

[4]. Xiong Xuhong, Li Quanrong. The claim for the period and extension cost of international contracted projects under FIDICcontract conditions. Hydropower station design, 2013, (01): 97103.

[5]. He Bosen, Zhang Shui Bo. Claim for Contractor under the conditions of construction contract of 1999 FIDIC. International economic cooperation, 2000, (05): 39 42.

[6]. Shan Jian. Analysis of the cause of construction claims and basic treatment principles. Chongqing: Chongqing University, 2004.

[7]. Liang Hongsheng. Analysis of the cost claims after the extension of the contract. International economic cooperation, 2009, (11): 75 77.

[8]. Justin S. Contractor Post Completion Claims Advice to Engineers. Journal of Profess- ional 155use in Engineering Duration and practice, 2004, VL3o (4):295 30. 